\title{
Iodixanol Pharmacokinetics in Children
}

\author{
W.H. Johnson, Jr., ${ }^{1}$ T.R. Lloyd, ${ }^{2}$ B.E. Victorica, ${ }^{3}$ V.R. Zales, ${ }^{4}$ M.L. Epstein, ${ }^{5}$ R.D. Leff, ${ }^{6}$ \\ R.H. Ardinger, Jr., ${ }^{7}$ T.L. Slovis, ${ }^{8}$ J.A. Johnson, ${ }^{9}$ P.A. Marsters ${ }^{9}$ \\ ${ }^{1}$ Department of Pediatrics, LM Bargeron Division of Pediatric Cardiology, University of Alabama at Birmingham, \\ Birmingham, AL 35294, USA \\ ${ }^{2}$ Department of Pediatrics, Division of Pediatric Cardiology, University of Michigan Medical Center, CS Mott Children's \\ Hospital, Ann Arbor, MI 48109, USA \\ ${ }^{3}$ Department of Pediatrics, Division of Pediatric Cardiology, University of Florida, Gainesville, FL 32610, USA \\ ${ }^{4}$ The Deborah Heart and Lung Center, Browns Mills, NJ 08015, USA \\ ${ }^{5}$ Division of Pediatric Cardiology, Children's Hospital of Michigan, Detroit, MI 48201, USA \\ ${ }^{6}$ Departments of Pharmacy and Pediatrics, The University of Kansas Medical Center, Kansas City, KS 66160, USA \\ ${ }^{7}$ Department of Pediatrics, Division of Pediatric Cardiology, The University of Kansas Medical Center, \\ Kansas City, KS 66160, USA \\ ${ }^{8}$ Department of Pediatric Imaging, Children's Hospital of Michigan, Detroit, MI 48201, USA \\ ${ }^{9}$ Nycomed Inc., Wayne, PA 19087, USA
}

\begin{abstract}
The objective of this report was to study the elimination pharmacokinetics of iodixanol in children. Iodixanol (VISIPAQUE®, Nycomed Inc., Wayne, PA, USA) is a new iso-osmolar iodinated radiocontrast agent. We hypothesized that elimination of this agent would be dependent on age-related differences in renal clearance. Seven centers enrolled 43 patients. Cardiac catheterization was performed in 41 patients and cranial computed tomography in 2. Patients were entered into 5 age groups: newborn to $<2$ months, 2 to $<6$ months, 6 months to $<1$ year, 1 to $<3$ years, and 3 to $\leq 12$ years. Plasma samples were obtained before and at 4 time periods after completion of iodixanol injection: $0.75-1.25$ hours, 2-4 hours, 8-12 hours, and 16-32 hours. Plasma concentrations of iodixanol were determined using high performance liquid chromatography. The primary pharmacokinetic endpoint was the terminal elimination rate constant $\left(k_{\mathrm{el}}\right)$. Data were complete for 40 patients, showing mean $k_{\mathrm{el}}$ significantly lower in newborn to <2-month-old patients vs older patients: $\left(\mathrm{h}^{-1}\right.$, mean \pm standard deviation [SD]): newborn to $<2$ months $0.185 \pm 0.060,2$ to $<6$ months, $0.256 \pm 0.046,6$ months to $<1$ year $0.299 \pm$ $0.042,1$ to $<3$ years $0.322 \pm 0.058$, and 3 to $\leq 12$ years $0.307 \pm 0.071$; with increasing age, excretion is more rapid, as shown by each age group's elimination half-life $\left(t_{1 / 2} \beta\right)(\mathrm{h}) 4.14 \pm 1.41,2.79 \pm 0.55,2.36 \pm 0.37,2.23 \pm$ 0.51 , and $2.36 \pm 0.52$, respectively. Iodixanol has elimi-
\end{abstract}

Correspondence to: W.H. Johnson, Jr., 320 Hillman Building, 620 South 20th Street, Birmingham, AL 35294, USA; email: wjohnson@ uab.edu nation in children $>6$ months of age that is comparable to normal adults. Prolonged elimination in children $<6$ months of age is related to renal immaturity.

Key words: Angiography — Contrast media - Pharmacokinetics

Iodixanol (VISIPAQUE® Injection) is a dimeric, nonionic radiographic contrast medium, which, unlike traditional ionic and nonionic contrast agents, has an osmolality equal to blood. Iodixanol may offer advantages over conventional hypertonic media, particularly in children with renal and myocardial immaturity. The elimination of iodixanol in children has not been previously studied.

Clearance of contrast agents in adults occurs primarily by renal elimination [9]. Patients with renal impairment have delayed elimination and prolonged plasma levels of contrast agents, including iodixanol, that are linearly related to the glomerular filtration rate (GFR) [5]. We hypothesized that the same would be true of infants (aged 0-6 months), in whom normal renal function, as assessed by GFR, is less than adult values [8]; this would result in slower renal elimination of iodixanol and lower terminal elimination rate constants $\left(k_{\mathrm{el}}\right)$. This would also show that $k_{\mathrm{el}}$ in older children would be similar to those reported for adults.

We performed a multicenter study to test this hypothesis by determining the pharmacokinetic profile of iodixanol in pediatric patients undergoing contrastenhanced diagnostic procedures. 


\section{Materials and Methods}

\section{Study Centers}

Seven pediatric centers participated: the University of Alabama at Birmingham, Birmingham, AL, USA; CS Mott Children's Hospital, Ann Arbor, MI, USA; the University of Florida, Gainesville, FL, USA; the Deborah Heart and Lung Center, Browns Mills, NJ, USA; Children's Hospital of Michigan (the Division of Pediatric Cardiology and the Department of Pediatric Imaging operated as separate entities), Detroit, MI, USA; and the University of Kansas Medical Center, Kansas City, KS, USA.

Authors JAJ and PAM were employed by the study sponsor, Nycomed, Inc. Other than the support provided by Nycomed, Inc. to each center for conducting this study, the other authors have no commercial interest in Nycomed, Inc.

\section{Patients}

Newborn infants ( $\geq 36$ weeks gestational age and $\geq 2000$ g body weight at time of enrollment) to children $\leq 12$ years of age, referred for an iodinated contrast-enhanced intravascular diagnostic procedure, were eligible. Patients undergoing interventional procedures (biopsy, balloon atrial septostomy, coil embolization, balloon dilation of aortic or pulmonary valves, or coarctation) were included.

Prior approval by each center's human subjects Institutional Review Board was received, and written informed consent was obtained from the parent or legal representative of each patient. No incentives were offered; iodixanol was provided by Nycomed at no cost.

\section{Radiographic Use}

Iodixanol (5,5' -[(2-hydroxy-1,3-propanediyl)bis(acetylimino)]bis[N, $\mathrm{N}^{\prime}$-bis(2,3-dihydroxypropyl)-2,4,6-triiodo-1,3-benzenedicarboxamide]) was supplied by Nycomed Inc. (Wayne, PA, USA) as a $652 \mathrm{mg} / \mathrm{ml}$ solution, equivalent to $320 \mathrm{mgI} / \mathrm{ml}$. Patients received iodixanol in identical fashion to approved contrast agents, without modification of dose or administration method. Pharmacokinetic samples were obtained at baseline (preinjection) and at four time periods after injection: $0.75-1.25$ hours, 2-4 hours, $8-12$ hours, and 16-32 hours. Blood samples were collected in potassium oxalate/sodium fluoride Vacutainer ${ }^{\mathrm{TM}}$ tubes (Becton Dickinson, Rutherford, NJ, USA) and were spun immediately. The plasma was separated, was frozen to $\leq-20^{\circ} \mathrm{C}$, and was shipped to Nycomed Inc. for analysis.

\section{Iodixanol Assay}

Plasma iodixanol assays were performed by Nycomed Inc. using sizeexclusion, high-performance liquid chromatography with ultraviolet detection [4]. The assays were modified as follows: reference standard concentrations ranged from $1.5-1000 \mu \mathrm{g} / \mathrm{ml}$. The assay was validated to a minimum quantifiable level of $1.5 \mu \mathrm{g} / \mathrm{mL}$. Triplicate qualitycontrol samples at three concentrations were included in each analytical run.

\section{Pharmacokinetic Analysis}

Based on data for adults, the concentration vs time curve is best estimated by a one-compartment model with a first-order elimination rate.
In adults, the distribution phase for intravascular administration is of small magnitude and occurs rapidly, so this term was assumed to be zero. The terminal elimination rate constant $\left(k_{\mathrm{el}}\right)$ was computed by linear regression of the natural logarithm of the plasma iodixanol concentration as a function of time:

$$
\mathrm{c}(\mathrm{t})=\mathrm{c}_{0} \mathrm{e}^{-\mathrm{kelt}^{\mathrm{e}}}
$$

where $\mathrm{c}(\mathrm{t})$ is the concentration at time $\mathrm{t}, \mathrm{c}_{0}$ is the concentration at time 0 , and $t$ is the time after the end of the administration of iodixanol (defined as time zero). Preinjection sample times were set to zero and actual times were used for all postinjection samples. Elimination halflife was calculated as $\mathrm{t}_{1 / 2}=0.693 / k_{\mathrm{el}}$.

\section{Study Design and Statistical Analysis}

Male and female patients were enrolled by age into 5 age groups: birth to $<2$ months, 2 to $<6$ months, 6 to $<12$ months, 1 to $<3$ years, and 3 to $\leq$ to 12 years. Analysis of interinstitutional differences was not performed because no more than 10 patients were enrolled per center.

The relationship between $k_{\mathrm{el}}$ and age for individual patients was modeled using a linear-plateau model $[2,6]$ (details available from the authors). $k_{\mathrm{el}}$ was analyzed for dose (continuous) and age-group (class) effects using a general linear model, because the dose of iodixanol varied from patient to patient in each group. Sample size was selected with the expectation that $k_{\mathrm{el}}$ in the youngest and oldest age groups could be distinguished from one another, based on published renal elimination rates [8]. We chose age-group intervals corresponding to these published data. Statistical tests were performed with a level of significance set at 0.05 .

\section{Results}

\section{Patient Demographics}

Forty-three patients were enrolled, ranging from 2-10 patients per center. All patients completed the trial. The data from 40 patients were used in the pharmacokinetic analyses. Two patients had incomplete pharmacokinetic sampling data; their pharmacokinetic data were excluded. Two additional patients were enrolled as replacements in the same respective age groups. A third patient was excluded from pharmacokinetic analyses because of abnormal renal function before participation in the trial. One patient, entered in error as the ninth patient in the 6 month to $<1$ year of age group, was included in the analyses.

The majority of the patients were boys $(63 \%)$ and Caucasian $(60 \%)$; the median age was 0.56 years (range 0-10.4) and median body weight $6.6 \mathrm{~kg}$ (range 3-40) (Table 1$)$. The majority of patients $(56 \%)$ had more than one diagnosis; the most common was ventricular septal defect $(40 \%)$, followed by patent ductus arteriosus (19\%), aortic stenosis (16\%), tetralogy of Fallot (14\%), atrial septal defect (14\%), pulmonary stenosis (14\%) and aortic hypoplasia (12\%). 
Table 1. Patient demographics and iodixanol dose

\begin{tabular}{|c|c|c|c|c|c|c|}
\hline Patient group & $<2$ months & 2 to $<6$ months & 6 to $<12$ months & 1 to $<3$ years & 3 to $\leq 12$ years & All patients \\
\hline Number of patients & 8 & 9 & 10 & 8 & 8 & 43 \\
\hline \multirow[t]{2}{*}{ Age } & 2 days & 0.29 years & 0.67 years & 1.84 years & 6.46 years & 0.56 years \\
\hline & 12 hours-59 days & $0.17-0.41$ years & $0.51-0.90$ years & $1.06-2.96$ years & $3.16-10.41$ years & $0-10.41$ years \\
\hline \multirow[t]{2}{*}{ Weight $\mathrm{kg}$} & 4 & 4.9 & 7.3 & 11.3 & 19.1 & 6.6 \\
\hline & $3-5$ & $3-6$ & $6-11$ & $7-14$ & $14-40$ & $3-40$ \\
\hline \multirow[t]{2}{*}{ Dose $\mathrm{gI} / \mathrm{kg}^{\mathrm{a}}$} & 1.5 & 1.5 & 1.3 & 1.2 & 0.8 & 1.3 \\
\hline & $0.7-3.2$ & $1.0-2.9$ & $0.8-2.7$ & $0.3-3.0$ & $0.3-1.3$ & $0.3-3.2$ \\
\hline \multirow[t]{2}{*}{ Dose volume $\mathrm{ml} / \mathrm{kg}$} & 4.8 & 4.8 & 4.2 & 3.9 & 2.4 & 4.0 \\
\hline & $2.0-10.0$ & $3.3-9.0$ & $2.4-8.5$ & $1.1-9.2$ & $1.0-4.0$ & $1.0-10.0$ \\
\hline \multirow[t]{2}{*}{ Number of injections } & 2.5 & 3 & 3 & 3 & 2 & 2 \\
\hline & $1-5$ & $2-5$ & $1-4$ & $1-8$ & $1-2$ & $1-8$ \\
\hline
\end{tabular}

Data are median and range.

${ }^{a} \mathrm{~g}$ iodine $/ \mathrm{kg}$ body weight.

\section{Type of Study, Dose, and Mode of Iodixanol Delivery}

Two patients who underwent head and/or neck CT scanning received intravenous iodixanol, whereas all other patients had injection during angiocardiography. The mean $( \pm \mathrm{SD})$ dose of iodixanol $320 \mathrm{mgI} / \mathrm{ml}$ was $1.49 \pm$ $0.75 \mathrm{gI} / \mathrm{kg}$ and the mean total volume was $4.67 \pm 2.34$ $\mathrm{ml} / \mathrm{kg}$. Median and range of dose data are presented in Table 1 . The mean duration of the procedure was $30 \pm 29$ minutes (range 0-102) and the median number of injections was 2 (range 1-8).

The median dose per $\mathrm{kg}$ of iodixanol $320 \mathrm{mgI} / \mathrm{ml}$ and median total volume per $\mathrm{kg}$ was similar for the four patient groups less than 3 years of age. The median dose per $\mathrm{kg}$ and median total volume per $\mathrm{kg}$ for patients $\geq 3$ years of age were approximately half that for patients $<3$ years of age (Table 1). Patients $<3$ years of age received an average of approximately 3 injections per patient; patients $\geq 3$ years of age averaged 1.6 injections per patient.

\section{Concurrent Medications and Drug Interaction}

Concurrent medications (from 24 hours before the start of the radiographic procedure until up to 32 hours postinjection) were received by $36(84 \%)$ of patients; the majority $(63 \%)$ received cardiovascular medications (e.g., digoxin, diuretics). Premedication/procedural medications were received by all patients. These medications included sedatives, anesthetics, and analgesics by $98 \%$ of patients, cardiovascular drugs (e.g., inotropes, vasodilators, prostaglandin) by $74 \%$ of patients, and antithrombotic drugs and/or intravenous solutions by $61 \%$. No drug interactions were identified.

\section{Pharmacokinetics}

The highest plasma iodixanol levels were found during the first sampling period (0.75-1.25 hours) after injec- tion, and ranged from $970-11,102 \mu \mathrm{g} / \mathrm{ml}$, with a median of $4718 \mu \mathrm{g} / \mathrm{ml}$.

The mean $k_{\mathrm{el}}$ was significantly lower in the newborn to $<2$ month age group than in any of the three oldest age groups ( $p \leq 0.0001)$. Mean $k_{\mathrm{el}}$ for the 2 to $<6$ month age group $(0.256 / \mathrm{h})$ was intermediate between that of the newborn to $<2$ month age group $(0.185 / \mathrm{h})$ and those of older patients $(0.299 / \mathrm{h}$ to $0.322 / \mathrm{h})$. Total dose was not a significant independent variable $(p=0.08)$. The mean $k_{\mathrm{el}}$ in the youngest age group $(0.185 / \mathrm{h})$ represented approximately $55 \%$ of the adult mean $k_{\text {el }}$ value of $0.336 / \mathrm{h}$ [1] (Table 2).

A segmented linear regression model (Fig. 1) showed increasing $k_{\mathrm{el}}$ from birth to approximately age 0.55 years ( $95 \%$ CI: 0.36 to 0.75 years). The model also showed estimated $k_{\mathrm{el}}$ for patients over 0.55 years as $0.313 / \mathrm{h}$ (corresponding to an elimination $\mathrm{t}_{1 / 2}$ of $2.21 \mathrm{~h}$ ). The predicted $k_{\mathrm{el}}$ at birth was $0.169 / \mathrm{h}$ (95\% CI: 0.131 to $0.208 / \mathrm{h}$, or a $\mathrm{t}_{1 / 2}$ of $4.1 \mathrm{~h}$ ). This model accounted for almost $97 \%$ of the observed age-related variability in $k_{\mathrm{el}}$.

\section{Laboratory Data}

Preinjection serum creatinine values ranged from 0.1-1.1 $\mathrm{mg} / \mathrm{dl}$ and did not correlate with age. Renal function was considered normal-for-age in all but the one patient who was excluded from the analyses. One day postinjection, 2 patients had increases in serum creatinine to levels above the $95 \%$ CI for age, yet no creatinine value exceeded $1.1 \mathrm{mg} / \mathrm{dl}$ in any patient. No patient showed clinical evidence of renal insufficiency, except for the patient excluded because of prexisting renal insufficiency. No patient had evidence of structural renal abnormalities.

\section{Discussion}

Pharmacokinetic data regarding contrast media in children is limited $[3,7]$. However, the pharmacokinetic pro- 
Table 2. Iodixanol elimination rate constant $\left(k_{\mathrm{el}}\right)$ and elimination halflife $\left(\mathrm{t}_{1 / 2}\right)$ by age group

\begin{tabular}{|c|c|c|c|c|c|}
\hline \multirow[b]{2}{*}{ Age group } & \multirow[b]{2}{*}{$N$} & \multicolumn{2}{|c|}{$k_{\mathrm{el}}\left(\mathrm{h}^{-1}\right)$} & \multicolumn{2}{|c|}{$t_{1 / 2}(h)$} \\
\hline & & Mean & $\mathrm{SD}$ & Mean & SD \\
\hline Newborn to $<2$ months & 8 & 0.185 & 0.060 & 4.14 & 1.41 \\
\hline 2 to $<6$ months & 8 & 0.256 & 0.046 & 2.79 & 0.55 \\
\hline 6 months to $<1$ year & 9 & 0.299 & 0.042 & 2.36 & 0.37 \\
\hline 1 to $<3$ years & 7 & 0.322 & 0.058 & 2.23 & 0.51 \\
\hline 3 to $\leq 12$ years & 8 & 0.307 & 0.071 & 2.36 & 0.52 \\
\hline
\end{tabular}

files of several iodinated radiographic contrast agents are well described in adults $[5,9,10,11]$. In a single-dose study of iodixanol administered to 40 healthy adult men [9], approximately $97 \%$ of iodixanol was excreted unchanged in the urine within 24 hours. Elimination was independent of dose and was similar to other nonionic agents.

We found iodixanol elimination rates in children older than 6 months ( $t_{1 / 2} \beta 2.32$ hours), similar to those reported for healthy adults ( $\mathrm{t}_{1 / 2} \quad \beta \quad 2.05$ hours) [9]. In adults with renal impairment, renal elimination of iodixanol and iohexol is via glomerular filtration only [5]. In our patients $<2$ months of age, we demonstrated prolonged clearance of iodixanol. This corresponds to this age group's lower, yet normal-for-age, GFR as published [8]. Infants in the 2 to 6-month age group had elimination intermediate between our youngest infants and patients older than 6 months. Our data confirm our hypothesis of an age-dependent renal elimination of iodixanol.

Our findings suggest that in pediatric patients, the renal elimination of iodixanol should be similar to conventional contrast media. We speculate that safety of iodixanol in children should not be related to its elimination pharmacokinetics.

In adult patients, clinical trials of iodixanol demonstrated similar efficacy, safety, and pharmacokinetics as compared with other nonionic agents [1]. Iodixanol solution is hypotonic at iodine concentrations adequate for imaging. Sodium and calcium are added to obtain a plasma-isoosmolal solution. The presence of sodium and calcium ions in iodixanol, and its osmolality, may result in fewer side effects. However, higher viscosity, as compared with other agents, may be a relative disadvantage.

More experience is needed in infants and children to determine if the chemical properties of iodixanol confer safety advantages over conventional nonionic agents.

\section{Study Limitations}

Our study is limited by the absence of a comparison group of pediatric patients receiving a conventional, nonionic agent. Although our patients had normal indicators of renal function for age, we did not measure creatinine clearance or GFR. The performance of these studies in pediatric patients is no less problematic today than when the widely accepted renal clearance data, upon which we based our hypotheses, were first measured decades ago [8]. It is possible that concurrent drugs could have altered iodixanol clearance by altering renal blood flow (e.g., inotropes) or by a direct effect on renal filtration (e.g., prostaglandin). However, our patient numbers were too small for analysis of a specific drug's possible effect. Although we found no relation of iodixanol dose to elimination rate, it is possible that a dose effect might be evident in the youngest patients, given a larger sample size.

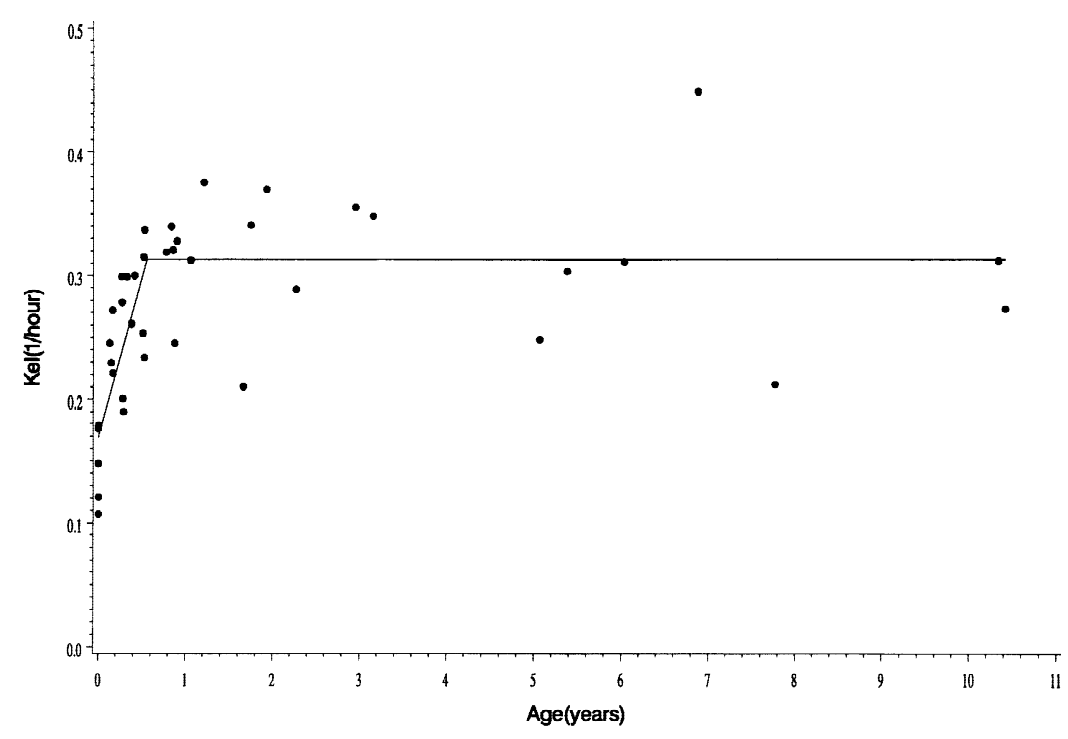

Fig. 1. Analysis of $k_{\mathrm{el}}$ vs age for iodixanol. The terminal elimination rate constant, $k_{\mathrm{el}}\left(\mathrm{h}^{-1}\right)$ is plotted against age (years) for each individual patient (closed dots). Using a segmented linear regression model, $k_{\mathrm{el}}$ increases with increasing age from birth to a value of $0.313 / \mathrm{h}$ at 0.55 years, according to the regression $k_{\mathrm{el}}=0.169+0.26(\mathrm{age})$; at ages older than 0.55 years, $k_{\mathrm{el}}$ is relatively constant, represented by a horizontal line with y-intercept of $0.313 / \mathrm{h}$. 


\section{Conclusion}

Our study demonstrates that iodixanol injection (320 $\mathrm{mgI} / \mathrm{ml}$ ), when used as a radiographic contrast agent in pediatric patients undergoing angiography or CT scanning, is eliminated in a fashion similar to that in adults. The elimination is dependent on the age-related degree of renal maturity.

Acknowledgments. We would like to acknowledge the valuable assistance of Connie S. Nixon, RN, Tandaw E. Samdarshi, MD, and Ronald W. Palmer, BS, MS. Funded in part by Nycomed Inc., 466 Devon Park Drive, Wayne, PA 19087-8630, USA.

\section{References}

1. Fountaine H, Harnish P, Andrew E, Grynne B (1996) Safety, tolerance, and pharmacokinetics of iodixanol injection, a nonionic, isosmolar, hexa-iodinated contrast agent. Acad Radiol 3:S475S484

2. Hudson DJ (1966) Fitting segmented curves whose join points have to be estimated. J Amer Statist Ass. 61:1097-1129

3. Klingmüller V, Brockmeier D, Schuster W, von Hattingberg HM (1983) Pharmakokinetische aspekte der ausscheidungsurographie im kindesalter. [Pharmacokinetic aspects of excretory urography in children]. ROFO Fortschr Geb Rontgenstr Nuklearmed 138:316322

4. Nomura H, Teshima E, Hakusui H (1991) Simple isocratic highperformance liquid chromatographic method for measurement of iodixanol in human plasma. J Chromatogr 572:333-338

5. Nossen JO, Jakobsen JA, Kjaersgaard P, Andrew E, Jacobsen PB, Berg KJ (1995) Elimination of the non-ionic X-ray contrast media iodixanol and iohexol in patients with severely impaired renal function. Scand J Clin Lab Invest 55:341-350

6. Ralston ML, Jennrich RI (1978) Dud, a derivative-free algorithm for nonlinear least squares. Technometrics 20:7-14

7. Stake G, Monclair T (1991) A single plasma sample method for estimation of the glomerular filtration rate in infants and children using iohexol, I: establishment of a body weight-related formula for the distribution volume of iohexol. Scand J Clin Lab Invest 51:335-342

8. Stewart CF, Hampton EM (1987) Effect of maturation on drug disposition in pediatric patients. Clin Pharm 6:548-564

9. Svaland MG, Haider T, Langseth-Manrique K, Andrew E, Hals PA (1992) Human pharmacokinetics of iodixanol. Invest Radiol 27: 130-133

10. Svaland MG, Kolmannskog PE, Lillevold PE, Nordal KP, Ressem L, Berg KJ (1992) Pharmacokinetics of iopental in patients with chronic renal failure. Acta Radiologica 33:482-484

11. Thomsen HS, Vestergaard A, Nielsen SL, Fogh-Andersen N, Golman K, Dorph S (1991) Renal clearance of an ionic high-osmolar and a nonionic low-osmolar contrast medium. Invest Radiol 26: $564-568$ 